However, for a long time in past there were no detected the positive correlations between the characteristics of sunspots cycle (Wolf number or the total area of spots) and polar magnetic flux (which characterized the value of poloidal field $B_{p}$ ) at the end of the cycle. In the terms of dynamo theory it was supposedly evidenced of the absence of functional dependence poloidal fields $B_{P}$ at the end of cycles on the toroidal field $B_{T}$ in maximum of cycles. As it turned out later, this was due to the fact that the surface $\alpha$-effect of Babcock-Leighton (which defined by tilt angles of the bipolar magnetic fields, turbulent diffusion and meridional circulation, and causes the regeneration of the poloidal field) is characterized by random fluctuations in time and space. The situation, however, changed drastically after the introducing of the parameter of magnetic strength spots of cycle, which is a product of the area of spots cycle and tilt angles of the bipolar magnetic groups. By combining assimilation of the area of spots, the distance between the weighted canters of opposite magnetic polarity and the tilt angles, the functional dependence of polar magnetic flux (which is an indicator of poloidal field $B_{P}$ ) on the assimilated parameter magnetic strength of sunspots (which describes the relative intensity of the toroidal field $B_{T}$ ) was restored. Within the framework of the $\alpha \Omega$-dynamo this indicates that the surface $\alpha$-effect of Babcock-Leighton leads to the generation of the poloidal magnetic field at the end of the current cycle, and assimilated parameter of magnetic strength spots of cycle is an integral component for future forecasts of solar activity based on the dynamo models.

УДК 524.7

E. Fedorova', Ph. D., A. Vasylenko², Ph. D., V. Zhdanov' ${ }^{1}$, Dr. Sci., Prof., ${ }^{1}$ Astronomical Observatory of National Taras Shevchenko University of Kyiv, Kyiv ${ }^{2}$ Main Astronomical Observatory of the NAS of Ukraine, Kyiv

\title{
PECULIAR AGNS FROM THE INTEGRAL AND RXTE DATA
}

We analysed how the active galactic nucleus (AGN) X-ray primary continuum depends on AGN activity in radio, using the data of RXTE Spectral \& Timing Database (Rossi X-ray Timing Explorer) and INTEGRAL (INTErnational Gamma-Ray Astrophysics Laboratory). Our aim is to test the relation between spectral shapes of these AGNs and the prediction of the "spinparadigm" model of the AGN "central engine". We have found that for the major part of radio-quiet (RQ) AGNs the value of highenergy exponential cut-off in primary spectrum appears to be significantly higher than $100 \mathrm{keV}$ and thus follows the "spinparadigm" predictions. In the same time, near $25 \%$ of radio-loud (RL) AGNs demonstrate the high-energy cut-off at the energies above $150 \mathrm{keV}$, contradicting the "spin paradigm". We have composed a sample of "peculiar" 69 RQ and 10 RL AGNs that seem to contradict to the "spin paradigm" for further individual studies of these objects.

Key words: galaxies: Seyfert - X-rays: galaxies: active - galaxies

Introduction. One of the most prominent physical differences between AGNs of various classes can be related with jet activity and RL/RQ dichotomy. There are several schemes explaining physical (non-geometrical) differences in the AGN structure, and one of the most well-known of them is often referred to as the "spin-paradigm". This model associates the jet activity with high values of the central super massive black hole (SMBH) spin as well as with the direction of rotation of the accretion disk (AD) around the black hole [1]. Namely, high values of the SMBH spin $(a>0.75)$ in prograde system (i.e. when the directions of the SMBH spin and the angular velocity of accretion disk rotation are the same) and retrograde systems (i.e. systems with SMBH spin antiparallel to the angular velocity of $\mathrm{AD}$ ) with the spin of SMBH a<-0.1 correspond to powerful jet and radio emission in RL AGN with the radio loudness parameter $R=L_{\text {vradio }} / L_{\text {vopt }}>100$ (here $L_{\text {nradio }}$ and $L_{\text {vopt }}$ are monochromatic luminosities at $5 \mathrm{GHz}$ and in $\mathrm{V}$ band, correspondingly [2]). The comparably low values of SMBH spin $(-0.1<a<0.5)$ corresponds to RQ AGNs with low values of radio loudness parameter $(R<100,[2])$ and weak or no jets[3].

Following this model, in RQ AGNs fit into standard Shakura-Synyaev AD model [4] (i.e. geometrically thin, radiatively efficient, steady state disk with zero viscosity at the sonic point) with almost radial infall inside the innermost stable orbit; whereas the disks in RL AGNs are "torqued" (there is nonzero magnetic viscosity at the sonic point) magnetized disks described by Agol and Krolik [5]. In such systems the spin energy of central SMBH can dissipate through the sonic point into the disk due to magnetic reconnection between the inner part of $A D$ and SMBH horizon. One of the consequences of this model is that the innermost part of $A D$ in RL $A G N$ is disrupted and smeared away by powerful centrifugally-driven jet outflows, leading to the low values of the exponential cutoff in the spectrum of the primary emission (below $100 \mathrm{keV}$ ), whereas the high values or absence of the high-energy cut-off are prescribed to RQ AGN spectrum. However, some objects were found which seem to contradict this paradigm [6-9]. That is why it is interesting to compare the high-energy exponential cut-off with the RL/RQ characteristics of AGNs.

In the present paper we obtain main parameters of the hard X-ray spectra $(0.5-250 \mathrm{keV})$ of 79 non-blazar Seyfert galaxies from RXTE spectra \& timing database to find the objects with peculiar spectral shape. Additionally, we consider for this aim the objects of INTEGRAL sample we worked out earlier in [10].

In Sections 2 we describe the samples of AGNs we considered, and describe the data and models, and present the best fit individual model parameters of the AGNs of RXTE sample.

In Section 3, we show the resulting subsample of peculiar AGNs from both RXTE and INTEGRAL samples. Finally, in the last section we discuss our results and draw out conclusions.

Sample, data and fitting. We compiled a sample of AGNs from the RXTE AGN spectra and timing database. After excluding blazars and bad datasets, the final sample consists of 79 AGNs, including $10 \mathrm{RL}$ and $69 \mathrm{RQ} A G N s$. We also included in our consideration the INTEGRAL sample of 95 AGNs we treated earlier in [10].

To perform our spectral fitting, we use the XSPEC 12.8 package of HEASOFT (High Energy Astrophysical SOFTware) software provided by NASA [11].

For the primary spectrum of AGN we used the standard power law with an exponential cut-off at high energies $A_{\mathrm{PL}}(E)=K E^{-\Gamma} \exp \left(-E / E_{c}\right)$ (cutoffpl), where $\Gamma$ is the photon index and $E_{c}$ is the cut-off energy. This primary emission is reflected from a neutral or ionized material of an accretion disc or gas-dust torus; to model it we used pexrav model [12]. This model describes a power-law emission partially reflected by an infinite flat slab of neutral or ionized medium. To take into account the proper absorption in the investigated object, described by the formula $A_{\text {abs }}(E)=\exp \left\{-N_{H} \sigma[E(1+z)]\right\}$ and 
modeled by zphabs model in XSPEC. Galactic absorption (in the Milky Way) is also included in all the spectral models, following the data by Kalberla et al.[13] and using the model phabs (the same as zphabs with the redshift $z=0$ ).

Thus the model in XSPEC for S1-S1.5 AGNs schematically looks like:

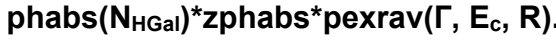

describing the emission of an AGN "central engine" both direct and reflection fractions (pexrav) with the same low level of absorption, and for S1.7 - S2 AGNs:

$\operatorname{phabs}\left(N_{H G a l}\right)^{*}\left(z p h a b s^{*}\right.$ cutoffpl($\left.\left(\Gamma, E_{c}, R\right)+\operatorname{pexrav}\left(\Gamma, E_{c}, R\right)\right)$,

describing the sum of strongly obscured direct emission of the "central engine" (cutoffpl) and slightly absorbed reflected one (pexrav).

For each galaxy of the sample, we obtained such spectral parameters as the power-law index, relative reflection parameter $R$, cut-off energy $E_{c}$ and intrinsic absorption value. The best-fit parameters are presented in Table 1, together with the errors, lower and upper limits at $90 \%$ confidence level. Below we perform also a correlation analysis of the main spectral parameters, namely "photon index - cut-off energy". The peculiar AGNs addressing the "spin-paradigm" are boldfaced and underlined in the Table 1. Objects with the best-fit value of the high-energy cut-off contradicting the "spin-paradigm" predictions, but with error's level too high to determine this finally (i. e. "candidates to the peculiar subsample"), are underlined only.

Best fit parameters of AGNs of RXTE sample

Table 1

\begin{tabular}{|c|c|c|c|c|c|}
\hline Source & Class & $\Gamma$ & $\mathrm{E}_{\mathrm{C}}, \mathrm{keV}$ & $\mathbf{R}$ & $\mathrm{N}_{\mathrm{H}}, 10^{22} \mathrm{~cm}^{-2}$ \\
\hline Mkn 335 & RQ NL S1 & $2.60 \pm 0.05$ & $>170$ & $4.6 \pm 2.7$ & $2.3 \pm 1.4$ \\
\hline RHS 03 (RBS 78) & RQ S1 & $1.37 \pm 0.12$ & $8_{-3}^{+18}$ & $\begin{array}{r}+1.5 \\
2.2_{-1.3}\end{array}$ & $<0.95$ \\
\hline Mkn 348 & RQ S2 & $1.77 \pm 0.07$ & $>100$ & $<7.0$ & $24 \pm 4$ \\
\hline PG 0052+251 & RQ S1.2 & $1.75 \pm 0.04$ & $\begin{array}{r}+42 \\
73_{-18}\end{array}$ & $<0.45$ & $<1.0$ \\
\hline TONS 180 & RQ NL S1 & $2.09 \pm 0.15$ & $34 \pm 21$ & $<0.3$ & $<0.5$ \\
\hline Fairall 9 & RQ S1 & $1.79 \pm 0.03$ & $>15$ & $3.8 \pm 0.3$ & $<1.0$ \\
\hline NGC 526A & RQ S1.9 & $1.25 \pm 0.05$ & $10 \pm 1$ & $4.2 \pm 0.7$ & $4100 \pm 400$ \\
\hline Mkn 590 & RQ S1.2 & $1.59_{-0.13}^{+0.14}$ & $\begin{array}{r}+9 \\
18_{-4}^{+9}\end{array}$ & $2.7_{-1.3}^{+1.8}$ & $<1.0$ \\
\hline NGC 1052 & RL S2 & $1.97 \pm 0.05$ & $\begin{array}{r}+9 \\
36_{-6}\end{array}$ & $24_{-5}^{+6}$ & $22 \pm 3$ \\
\hline NGC 1068 & RQ S2 & $1.62 \pm 0.12$ & $>1400$ & $<1.16$ & $>930$ \\
\hline $4 \cup 0241+61$ & RQ S1.2 & $1.94 \pm 0.05$ & $\begin{array}{c}{ }^{+189} \\
160_{-74}\end{array}$ & $1.21 \pm 0.26$ & $3.35 \pm 0.33$ \\
\hline RHS 17 & RQ S1 & $2.50 \pm 0.11$ & $>70$ & $18 \pm 5$ & $<1.0$ \\
\hline$\underline{4 \mathrm{U} 0241+611}$ & RQ S1.2 & $1.92 \pm 0.06$ & $76 \pm 64$ & $1.5 \pm 0.4$ & $3.2 \pm 0.4$ \\
\hline NGC 1386 & RQ S2 & $3.5 \pm 1.3$ & $>3$ & $<47$ & $2.0_{-0.8}^{+0.7}$ \\
\hline $3 \mathrm{C} 111$ & RL S1 & $1.88 \pm 0.01$ & $>1500$ & $0.16 \pm 0.05$ & $2.7 \pm 0.2$ \\
\hline $3 \mathrm{C} 120$ & RL S1 & $1.92 \pm 0.01$ & $230 \pm 40$ & $0.38 \pm 0.06$ & $0.45 \pm 0.33$ \\
\hline IRAS 04575-7537 & RQ S2 & $2.28 \pm 0.04$ & $15 \pm 1$ & $10 \pm 2$ & $\begin{array}{r}+26 \\
24-10\end{array}$ \\
\hline Ark 120 & RQ S2 & $2.07 \pm 0.04$ & $26 \pm 4$ & $3.5 \pm 0.4$ & $0.8 \pm 0.2$ \\
\hline Pictor A & RQ S1 & $1.35 \pm 0.03$ & $13 \pm 1$ & $1.0 \pm 0.3$ & $<1.0$ \\
\hline E253-G3 & RQ S2 & $3.18_{-1.9}^{+0.8}$ & $>15$ & $<35$ & $\begin{array}{r}+42 \\
151-37\end{array}$ \\
\hline NGC 2110 & RQ S2 & $2.09 \pm 0.05$ & $>500$ & $1.1 \pm 0.5$ & $24 \pm 2$ \\
\hline MCG+8-11-11 & RQ S1 & $1.40 \pm 0.04$ & $11 \pm 1$ & $4.8 \pm 0.6$ & $<1.0$ \\
\hline PKS 0558-504 & RQ NL S1 & $2.17 \pm 0.03$ & $>289$ & $<0.22$ & $<1.0$ \\
\hline Mkn 3 & RQ S2 & $1.71 \pm 0.06$ & $>300$ & $<3.3$ & $\begin{array}{r}+3.4 \\
8.8-3.3 \\
\end{array}$ \\
\hline Mkn 79 & RQ S1.2 & $1.95 \pm 0.03$ & $19_{-4}^{+30}$ & $8.7 \pm 0.9$ & $<0.9$ \\
\hline PG 0804+761 & RQ S1 & $2.12 \pm 0.23$ & $>20$ & $2.4 \pm 1.2$ & $<2.4$ \\
\hline PKS 0921-213 & RL S1 & $1.70 \pm 0.06$ & $\begin{array}{l}+1700 \\
132-66 \\
\end{array}$ & $\begin{array}{r}+0.42 \\
0.50_{-0.39}\end{array}$ & $<0.81$ \\
\hline Mkn 110 & RQ NL S1.5 & $1.79 \pm 0.02$ & $\begin{array}{r}+25 \\
52-13 \\
\end{array}$ & $0.98 \pm 0.01$ & $0.81 \pm 0.20$ \\
\hline NGC 2992 & RQ S2 & $2.00_{-0.09}^{+0.10}$ & $16_{-2}^{+3}$ & $12.6_{-7.8}^{+4.1}$ & $15_{-13}^{+14}$ \\
\hline MCG-5-23-16 & RQ S2 & $2.21 \pm 0.03$ & $>500$ & $0.7 \pm 0.2$ & $33 \pm 2$ \\
\hline NGC 3227 & RQ S1.5 & $1.20 \pm 0.03$ & $>1300$ & $1.9 \pm 0.3$ & $23_{-6}^{+5}$ \\
\hline NGC 3281 & $\mathrm{RQ}$ S2 & $2.6 \pm 0.4$ & $>30$ & $<4.0$ & $300_{-7}^{+9}$ \\
\hline NGC 3516 & RQ S1.5 & $2.14 \pm 0.06$ & $>209$ & $2.9 \pm 0.5$ & $21 \pm 3$ \\
\hline PG 1116+215 & RQ S1 & $2.35 \pm 0.11$ & $18 \pm 4$ & $23 \pm 7$ & $<0.5$ \\
\hline NGC 3783 & RQ S1 & $2.00 \pm 0.02$ & $900 \pm 400$ & $0.95 \pm 0.13$ & $<0.5$ \\
\hline
\end{tabular}


End of the table 1

\begin{tabular}{|c|c|c|c|c|c|}
\hline NGC 3998 & RQ S1 & $2.00 \pm 0.06$ & $106 \begin{array}{c}+690 \\
-50\end{array}$ & $<0.39$ & $<0.5$ \\
\hline NGC 4051 & RQ NL S1.5 & $2.59 \pm 0.01$ & $\begin{array}{r}+540 \\
397-146\end{array}$ & $7.1 \pm 0.3$ & $1.5 \pm 0.2$ \\
\hline PG 1202+281 & RQ S1 & $2.2 \pm 0.5$ & $>10$ & $0.88 \pm 0.09$ & $<0.5$ \\
\hline NGC 4151 & RQ S1.5 & $1.53 \pm 0.06$ & $112_{-8}^{+32}$ & $9.8 \pm 3.3$ & $9.8 \pm 0.3$ \\
\hline PG 1211+143 & RQ NL S1 & $2.41 \pm 0.05$ & $2{ }^{+11}$ & $3.9 \pm 1.2$ & $9.8 \pm 0.3$ \\
\hline Mkn 766 & RQ NL S1 & $2.12 \pm 0.09$ & $\begin{array}{r}+7 \\
23-4\end{array}$ & $>3.5$ & $<0.5$ \\
\hline$\underline{\text { NGC } 4258}$ & RQ S2 & $1.34 \pm 0.17$ & $\begin{array}{r}+4 \\
11-2\end{array}$ & $1.9 \pm 1.5$ & $<0.5$ \\
\hline NGC 4388 & RQ S2 & $2.15 \pm 0.19$ & $>500$ & $5.6 \pm 4.7$ & $6.4 \pm 0.4$ \\
\hline $\begin{array}{l}\text { TON 1542 (Mkn 771, } \\
\text { Ark 374, RBS 1125) }\end{array}$ & RQ S1 & $1.8 \pm 0.6$ & $10_{-4}^{+21}$ & $<1$ & $<0.5$ \\
\hline NGC 4507 & RQ S2 & $1.77 \pm 0.12$ & $>500$ & $0.57 \pm 0.05$ & $6.4 \pm 0.6$ \\
\hline NGC 4593 & RQ S1 & $1.82 \pm 0.01$ & $1130_{-471}^{+2291}$ & $4.2 \pm 0.8$ & $<0.5$ \\
\hline NGC 4945 & RQ S2 & $1.22 \pm 0.09$ & $143 \pm 44$ & $4.2 \pm 0.8$ & $3.2 \pm 0.2$ \\
\hline Centaurus A (NGC 5128) & RL S2 & $1.86 \pm 0.02$ & $>1000$ & $<0.0002$ & $12 \pm 1$ \\
\hline MCG-6-30-15 & RQ NL S1.2 & $2.42 \pm 0.01$ & $>2008$ & $3.1 \pm 0.1$ & $2.7 \pm 0.2$ \\
\hline IRAS 13349+2438 & RQ S1 & $1.7 \pm 0.8$ & $\begin{array}{l}+20 \\
8-3\end{array}$ & $<22$ & $<0.5$ \\
\hline IC 4329A & RQ S1.2 & $2.05 \pm 0.05$ & $>300$ & $0.83 \pm 0.09$ & $15 \pm 3$ \\
\hline Mkn 279 & RQ S1.5 & $1.7 \pm 0.1$ & $12 \pm 1$ & $4.4 \pm 1.3$ & $<0.5$ \\
\hline Circinus & RQ S2 & $3.25 \pm 0.05$ & $>2000$ & $>1000$ & $54 \pm 2$ \\
\hline NGC 5506 & RQ S1.9 & $2.3 \pm 0.1$ & $>800$ & $4 \pm 1$ & $13 \pm 3$ \\
\hline NGC 5548 & RQ S1.5 & $1.98 \pm 0.6$ & $287 \pm 118$ & $0.9 \pm 0.2$ & $14 \pm 2$ \\
\hline PG 1416-129 & RQ S1 & $1.4 \pm 0.2$ & $503_{-405}^{+3505}$ & $<4.5$ & $<0.5$ \\
\hline PG 1440+356 (Mkn 478) & RQ NL S1 & $\begin{array}{r}+0.6 \\
3.5-2.5\end{array}$ & $>5$ & $<38.3$ & $<0.5$ \\
\hline MCG-2-40-4 & RQ S2 & $1.8 \pm 0.7$ & $>7$ & $<6.8$ & $\begin{array}{r}+24 \\
80-16 \\
\end{array}$ \\
\hline NGC 6251 & RQ S2 & $2.27 \pm 0.15$ & $>60$ & $1.3 \pm 0.5$ & $680 \pm 120$ \\
\hline NGC 6240 & RQ S2 & $1.96 \pm 0.28$ & $>605$ & $5.1 \pm 0.6$ & $130 \pm 20$ \\
\hline NGC 6300 & RQ S2 & $2.41 \pm 0.09$ & $\begin{array}{r}+360 \\
354-250\end{array}$ & $>46$ & $\begin{array}{r}+13 \\
80_{-10}^{+10}\end{array}$ \\
\hline PDS 456 & RQ S1 & $3.8 \pm 0.2$ & $>200$ & $42 \pm 25$ & $<0.5$ \\
\hline 3 C 382 & RL S1 & $1.97 \pm 0.01$ & $>117$ & $0.39 \pm 0.07$ & $1.89 \pm 0.23$ \\
\hline IRAS 1825-5926 & RQ S2 & $1.9 \pm 0.6$ & $10 \pm 6$ & $<8.8$ & $5.2 \pm 1.8$ \\
\hline ESO 103-G35 & RL S2 & $2.33 \pm 0.02$ & $>70$ & $>40$ & $36 \pm 1$ \\
\hline$\underline{3 C} 390.3$ & RL S1 & $1.79 \pm 0.04$ & $126_{-37}^{+73}$ & $0.25 \pm 0.11$ & $1440 \pm 250$ \\
\hline$\frac{3 \mathrm{C} \mathrm{405}}{\text { (Cygnus A) }}$ & RL S2 & $2.81 \pm 0.13$ & $>1000$ & $<1.7$ & $46 \pm 1$ \\
\hline RHS 56 & RQ NL S1 & $2.4 \pm 0.4$ & $>41$ & $4.5 \pm 2.1$ & $<0.5$ \\
\hline Mkn 509 & RQ S1.2 & $1.91 \pm 0.01$ & $466_{-114}^{+211}$ & $0.51 \pm 0.05$ & $0.68 \pm 0.15$ \\
\hline IC 5063 & RQ S2 & $2.22 \pm 0.03$ & $>290$ & $28 \pm 12$ & $44 \pm 2$ \\
\hline NGC 7172 & RQ S2 & $2.04 \pm 0.02$ & $>540$ & $3.7 \pm 1.1$ & $25 \pm 12$ \\
\hline NGC 7213 & RL S1.5 & $1.59 \pm 0.08$ & $10 \pm 3$ & $4.9 \pm 2.0$ & $<0.01$ \\
\hline NGC 7314 & RQ S1.9 & $2.25 \pm 0.12$ & $>600$ & $1.9 \pm 0.5$ & $27 \pm 7$ \\
\hline Ark 564 & RQ NL S1 & $1.70 \pm 0.07$ & $\begin{array}{r}+7 \\
27_{-4}^{+} \\
\end{array}$ & $0.6 \pm 0.2$ & $12.2 \pm 0.3$ \\
\hline MR 2251-178 & RQ S1.5 & $1.78 \pm 0.01$ & $\begin{array}{r}+67 \\
225_{-43}\end{array}$ & $<0.03$ & $1.0 \pm 0.2$ \\
\hline NGC 7469 & RQ S1.2 & $2.1 \pm 0.1$ & $>800$ & $0.76 \pm 0.25$ & $3.0 \pm 0.4$ \\
\hline MCG-2-58-22 (Mkn 926) & RQ S1.5 & $1.90 \pm 0.07$ & $\begin{array}{c}+375 \\
303-50\end{array}$ & $0.39 \pm 0.15$ & $3.4 \pm 0.3$ \\
\hline NGC 7582 & RQ S2 & $2.35 \pm 0.09$ & $>728$ & $120 \pm 80$ & $50 \pm 9$ \\
\hline$\underline{\text { RHS } 61}$ & RQ S1 & $1.81 \pm 1.11$ & $\begin{array}{l}+40 \\
8-4\end{array}$ & $<14$ & $<1.0$ \\
\hline
\end{tabular}

The $\Gamma$ vs. $E_{c}$ diagram for both the objects of RXTE and INTEGRAL samples is shown on the Fig. 1. Correlations for parameters for the total set of the data are shown in the Table 2. 
Correlation coefficients between spectral parameters for the AGNs of RXTE sample

\begin{tabular}{|l|c|c|c|}
\hline Correlation, parameters -> & $\boldsymbol{\Gamma}, \boldsymbol{E}_{c}$ & $\boldsymbol{\Gamma}, \boldsymbol{R}$ & $\boldsymbol{E}_{c}, \boldsymbol{R}$ \\
\cline { 1 - 4 } Object class $\downarrow$ & 0.08 & 0.28 & 0.196 \\
\hline $\mathrm{RQ}$ & 0.58 & -0.09 & 0.26. \\
\hline $\mathrm{RL}$ & 0.19 & 0.26 & 0.14 \\
\hline all & & & 2 \\
\hline
\end{tabular}

As we can see we have small correlations between photon index and reflection parameter, as well as between the cutoff energy and reflection parameter, however, we see that the correlation between photon index and cut-off energy is considerable. At the same time, the mean values of these spectral parameters over the subsamples of $R L$ and $R Q A G N s$ do not differ within the error limits: $\Gamma=1.91 \pm 0.13$ and $E_{c}=296_{-37}^{+690} \mathrm{keV}$ for $\mathrm{RQ} A G N s$, and $\Gamma=1.73 \pm 0.08$ and $E_{C}=390_{-25}^{+1000}$ $\mathrm{keV}$ for RL ones.

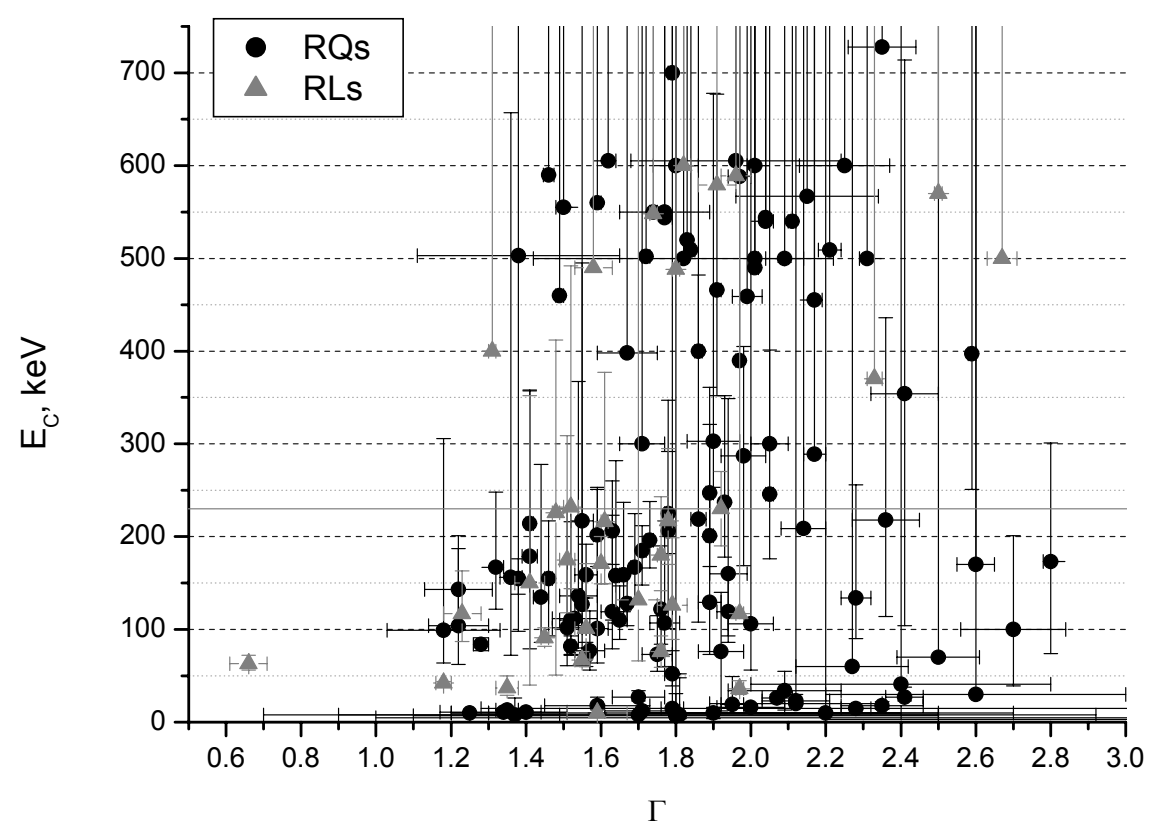

Fig. 1. Photon index vs. cut-off energy diagram for the AGNs of RXTE and INTEGRAL samples

Peculiar AGNs and spin-paradigm. Analysis performed had revealed the subsample of AGNs deviating from the "spinparadigm" prescriptions. The "mainstream" of RQ AGNs really follows this pattern, and only 25 objects from 150 (15\% of total number of $R Q$ objects) demonstrate the exponential high-energy cut-off below or near $100 \mathrm{keV}$. The situation with $\mathrm{RL}$ objects is opposite: only 17 of total 35 objects (48\%) have high-energy cut-offs below $100 \mathrm{keV}$ in their spectra. Additionally to the results of the Table.1, the peculiar AGNs found in the INTEGRAL sample are shown in the Table.3 (for these objects the same spectral model was applied in [10] to describe hard X-ray continuum emission above $3 \mathrm{keV}$ ). Here the "confirmed" peculiar AGNs with high enough accuracy level of parameters determining are also boldfaced. These sources should be recommended for further more detailed investigations of the full set of data available on them to understand exactly the reason of their contradiction with spin paradigm. Presumably, such high percentage of "spin-paradigm-deviating" AGNs among the RL party can be caused by jet contamination of a primary spectrum of the "central engine".

Conclusions. We have performed the spectral modelling of the RXTE data of 79 non-blazar AGNs from RXTE spectra \& timing database, to test the correspondence between their spectral properties and the pattern predicted by the "spinparadigm" model of AGN "central engine". We also used to this purpose the original sample of 95 AGNs observed by INTEGRAL mission which we have investigated earlier in [10].

As a result we have revealed $25 \mathrm{RQ}$ and $17 \mathrm{RL}$ AGNs demonstrating a behaviour contradicting the "spin-paradigm" prescribing low values of high-energy exponential cut-off in RL AGN spectra and higher ones for RQ AGNs. These objects have to be studied in details to clear out the reasons of such contradiction. For instance, the most probable reasons of the great percentage of peculiar RL object can be in fact "fake" caused by the X-ray contamination from the jet. However, in the same time we note, especially for $\mathrm{RQ}$ objects, that the percentage of peculiar objects can be a bit higher due to uncorrelated variability in different ranges of energies [15]: a part of objects with a variable spectral shape like NGC 4388 or NGC 4945 [7, 14], can be missed in an investigation like that of the present paper.

Acknowledgements. This research has made use of data and software obtained through the RXTE and INTEGRAL science data centers (ESA); provided by the NASA/Goddard Space Flight Center. The work has been supported by the scientific program "Astronomy and Space Physics" of Taras Shevchenko National University of Kyiv. 
Peculiar AGNs from RXTE and INTEGRAL samples. The dash in the last column (Ec) denotes the absence

Table 3. of high-energy exponential cut-off in th spectrum of an object (cut-off has not been detected)

\begin{tabular}{|c|c|c|c|c|}
\hline Name & Class & $\Gamma$ & $R$ & $E_{c}, \mathrm{keV}$ \\
\hline IGR J18027-1455 & RQ S1 & $1.52 \pm 0.03$ & $0.63_{-0.38}^{+0.68}$ & $110_{-38}^{+106}$ \\
\hline WKK 1263 & RQ S1 & $1.63 \pm 0.03$ & $1.51_{-0.60}^{+0.66}$ & $119_{-40}^{+104}$ \\
\hline GRS 1734-292 & RQ S1 & $1.52 \pm 0.01$ & $0.38_{-0.19}^{+0.15}$ & $82_{-9}^{+11}$ \\
\hline IGR J16482-3036 & RQ S1 & $1.59_{-0.02}^{+0.04}$ & $1.49_{-0.68}^{+1.09}$ & $101_{-37}^{+150}$ \\
\hline IGR J17488-3253 & RQ S1 & $1.44 \pm 0.02$ & $0.18 \pm 0.18$ & $135_{-49}^{+143}$ \\
\hline SWIFT J1038.8-4942 & RQ S1 & $1.51 \pm 0.04$ & $1.25 \pm 0.38$ & $102_{-41}^{+128}$ \\
\hline LEDA 090443 & RQ S1 & $2.28 \pm 0.04$ & $6.26_{-1.17}^{+1.36}$ & $134_{-44}^{+122}$ \\
\hline IGR J07597-3842 & RQ S1 & $1.56 \pm 0.01$ & $0.61_{-0.33}^{+0.35}$ & $71_{-11}^{+15}$ \\
\hline NGC 4593 & RQ S1 & $1.76 \pm 0.01$ & $0.69 \pm 0.11$ & $122_{-38}^{+68}$ \\
\hline 2E $1853.7+1534$ & RQ S1 & $1.89 \pm 0.03$ & $2.24_{-0.81}^{+0.93}$ & $129_{-56}^{+232}$ \\
\hline 1A $1343-60$ & RQ S1 & $1.65 \pm 0.02$ & $0.58 \pm 0.19$ & $110_{-21}^{+37}$ \\
\hline NGC 4151 & RQ S1 & $1.28 \pm 0.02$ & $0.05 \pm 0.03$ & $84 \pm 7$ \\
\hline M 87 & RL S1 & $2.50 \pm 0.01$ & 0 & - \\
\hline IGR J13109-5552 & RL S1 & $1.58 \pm 0.05$ & $1.58_{-1.15}^{+1.35}$ & - \\
\hline 3C 382 & RL S1 & $1.76 \pm 0.01$ & $0.68 \pm 0.19$ & $180_{-38}^{+63}$ \\
\hline 3C 111 & RL S1 & $1.60 \pm 0.01$ & $0.29 \pm 0.11$ & $171_{-22}^{+29}$ \\
\hline $3 C 390.3$ & RL S1 & $1.78 \pm 0.01$ & $1.28 \pm 0.17$ & $217_{-47}^{+78}$ \\
\hline Pictor A & RL S1 & $1.80 \pm 0.01$ & $0.99 \pm 0.23$ & - \\
\hline $3 C 120$ & RL S1 & $1.74 \pm 0.01$ & $0.25_{-0.09}^{+17.79}$ & - \\
\hline QSO B0241+62 & RL S1 & $1.61 \pm 0.01$ & $1.05 \pm 0.13$ & $216_{-67}^{+161}$ \\
\hline S5 2116+81 & RL S1 & $1.96 \pm 0.04$ & $2.37_{-0.99}^{+1.15}$ & - \\
\hline WKK 6471 & RL S1 & $1.91 \pm 0.05$ & 0 & - \\
\hline MCG-01-24-012 & RQ S2 & $1.77 \pm 0.04$ & $1.88_{-1.02}^{+1.22}$ & $107_{-47}^{+110}$ \\
\hline ESO 103-35 & RQ S2 & $1.94 \pm 0.03$ & $1.89_{-0.69}^{+0.82}$ & $119_{-26}^{+40}$ \\
\hline PGC 037894 & RQ S2 & $1.54_{-0.04}^{+0.06}$ & $1.42_{-1.04}^{+1.53}$ & $136_{-56}^{+231}$ \\
\hline WKK 0560 & RQ S2 & $1.22_{-0.07}^{+0.10}$ & $1.44_{-1.73}^{+2.97}$ & $104_{-42}^{+97}$ \\
\hline NGC 4138 & RQ S2 & $1.36 \pm 0.03$ & $0.09_{-0.01}^{+0.72}$ & $156_{-84}^{+501}$ \\
\hline NGC $1194^{1}$ & RQ S2 & $1.18 \pm 0.15$ & $1.15 \pm 0.89$ & $99_{-35}^{+207}$ \\
\hline ESO 506-27 & RQ S2 & $1.46 \pm 0.01$ & $0.73 \pm 0.34$ & $155 \pm 62$ \\
\hline IGR J20187+4041 & RQ S2 & $1.57 \pm 0.04$ & $1.48_{-1.07}^{+1.31}$ & $77_{-21}^{+43}$ \\
\hline IC 4518A & RQ S2 & $1.55 \pm 0.01$ & $2.62 \pm 0.61$ & $127_{-66}^{+663}$ \\
\hline NGC 4992 & RQ S2 & $1.41 \pm 0.02$ & 0 & $\begin{array}{r}+180 \\
179-100 \\
\end{array}$ \\
\hline NGC 6240 & RQ S2 & $2.70_{-0.16}^{+0.11}$ & 0 & $100_{-61}^{+101}$ \\
\hline NGC 1052 & RL S2 & $1.41 \pm 0.04$ & $0.10_{-0.05}^{+0.34}$ & $\begin{array}{r}+202 \\
150-110 \\
\end{array}$ \\
\hline Mrk 6 & RL S2 & $1.48 \pm 0.01$ & $0.52 \pm 0.13$ & $\begin{array}{r}+186 \\
226-175 \\
\end{array}$ \\
\hline 3C 405 & RL S2 & $1.51 \pm 0.02$ & 0 & $175_{-57}^{+113}$ \\
\hline NGC 1275 & RL S2 & $2.67 \pm 0.04$ & $0.59 \pm 0.39$ & - \\
\hline NGC 5128 & RL S2 & $1.82 \pm 0.02$ & 0 & - \\
\hline NGC 5252 & RL S2 & $1.31 \pm 0.01$ & 0 & - \\
\hline
\end{tabular}

References

1. Garofalo D., Evans D.A., Sambruna R.M. // MNRAS, 2010. - Vol. 406. - P. 975.

2. Sikora M., Stawarz L., Lasota J.-P. // http://www.slac.stanford.edu/cgi-wrap/getdoc/slac-pub-12284.pdf.

3. Garofalo D. // Adv. Astron., 2013. - ID 213105.

4. Shakura N. I., Sunyaev R. A. // A\&A, 1973. - Vol. 24. - P. 337.

5. Agol E., Krolik J.H. // Astron. J., 2000. - Vol. 528. - P. 161-170.

6. De Rosa A., Bassani L., Ubertini P. et al.// A\&A. - 2008. - Vol.483. - P. 749 
7. Fedorova E., Beckmann V., Neronov A., Soldi S.//MNRAS. - 2011. - Vol.417. - P.1140.

8. Soldi S., Beckmann V., Gehrels N., De Jong S., Lubinski P. // 2011, in: proc.of the Workshop "Narrow-Line Seyfert 1 Galaxies and Their Place in the Universe". ArXiv:1105.5993.

9. Soldi S., Beckmann V.,Bassani L., Courvoisier T.J.-L. et al. // A\&A, 2005. - Vol. 444. - P. 431

10. Vasylenko A., Zhdanov V., Fedorova E. // Astroph. Space Sci., 2015. - Vol. 360. - P. 71.

11. Arnaud K., Gordon C., Dorman B. An X-Ray Spectral Fitting Package // https://heasarc.gsfc.nasa.gov/xanadu/xspec/manual/manual.html.

12. Magdziarz P., Zdziarski, A. A. //MNRAS, 1995. - Vol. 273. - P. 837.

13. Kalberla P. M. W., Burton W. B., Hartmann D., Arnal E. M., Bajaja E., Morras R., Poppel W. G. L. // A\&A, 2005. - Vol. $440 .-$ P. 775

14. Fedorova E., Zhdanov V.I. // Kinemat.Phys.Celest.Bodies, 2016. - Vol. 32, is.4. - P. 172-180.

15. Chesnok N.G., Sergeev S.G., Vavilova I.B. // Kinemat. Phys. Celest. Bodies, 2009. - Vol. 25, N 2. - P. $107-113$.

Надійшла до редколегії 15.06.16

Федорова О., канд. фіз.-мат. наук,

Астрономічна обсерваторія

Київського національного університету імені Тараса Шевченка, Київ

Василенко А., канд. фіз.-мат. наук,

Головна астрономічна обсерваторія НАН України, Київ

Жданов В., проф.

Астрономічна обсерваторія

Київського національного університету імені Тараса Шевченка, Київ

\section{ПЕКУЛЯРНІ АЯГ ЗА ДАНИМИ INTEGRAL TA RXTE}

Проведено аналіз залежності спектральних параметрів первинного континууму активних ядер галактик від типу їх активності в радіодіапазон, використовуючі дані космічних місій INTEGRAL та RXTE з метою перевірки відповідності форми їх спектра до передбачень моделі будови центральної машини АЯГ "спін-парадігма". Е результаті виявлено, що для більшості РТ АЯГ значення експоненційного завалу на високих енергіях значно перевищує 100 кеВ, або навіть відсутнє, що відповідає передбаченням спін-парадигми, тоді як для РГ АЯГ блтзько 25 \% обєктів не вкладаються у спін-парадігму і мають експоненційний завал у спектрі на енергіях вище 150 кеВ, або ж не мають такого завалу зовсім. Складено вибірку таких об'єктів для подальшого детального дослідження.

Федорова Е., канд. физ.-мат. наук,

Астрономическая обсерватория

Киевского национального университета имени Тараса Шевченко, Киев;

Василенко А., канд. физ.-мат. наук,

Главная астрономическая обсерватория НАН Украины, Киев;

Жданов В., проф.,

Астрономическая обсерватория

Киевского национального университета имени Тараса Шевченко. Киев

\section{ПЕКУЛЯРНЫЕ АЯГ ПО ДАННЫM INTEGRAL И RXTE}

Проведен анализ зависимости спектральных параметров первичного континуума активных ядер галактик от типа их активности в радиодиапазоне, с использованием данных космических миссий INTEGRAL и RXTE, для проверки соответствия формы их спектров предсказаниям, сделанным на основе модели строения центральной машины АЯГ "спин-парадигма". В результате обнаружено, что для большинства РТ АЯГ значения экспоненциального завала на высоких энергиях сущестенно превишает 100 кеВ, или вообще отсутствует, в соотетствии с предсказаниями "спин-парадигмы", тогда как для РГ АЯГ около 25 \% объектов не соотвествуют "спин-парадигме", демонстрируя экспоненциальный завал в спектре на энергиях, превышающих 150 кеВ, или даже не имеют такового завала вообще. Собрано выборку таких объектов для дальнейшего более детального их исследования.

УдК 523.982

Кй̈вський національний університет імені Тй, д-р фіз.-мат. наук,

С. Осіпов, канд. фіз.-мат. наук,

Головна астрономічна обсерваторія НАН України, Київ

\section{ВИМІРЮВАННЯ МАГНІТНИХ ПОЛІВ У СОНЯЧНИХ ПЛЯМАХ ЗА СПЕКТРАЛЬНИМИ ЛІНІЯМИ 3 РІЗНИМИ ФАКТОРАМИ ЛАНДЕ}

Представлено результати прямих вимірювань магнітного поля в сонячних плямах, які спостерігались на горизонтальному сонячному телескопі АЦУ-5 ГАО НАН України у червні-липні 2015 р. Магнітні поля вимірювались за зеєманівським розщепленням кількох ліній Fel, Mnl ma Nil, розташованих у спектрі поблизу ліній Fel 5434.5 Á ma Fel 6093.66 Á. Ефективні фактори Ланде $g_{\text {eff }}$ цих ліній - у межах від -0.22 до 2.14. Виявлено суттєві відмінності виміряних напруже-

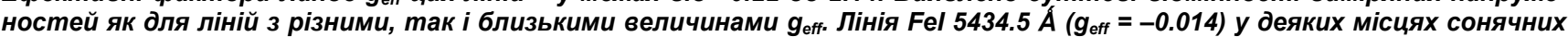
плям виявляє достовірне розщеплення, що відповідає магнітним полям напруженістю до $2.5 \mathrm{k} Г c$. У всіх досліджених випадках лінія $\mathrm{Fel} 6094.419$ Á з негативним фактором Ланде $\left(g_{\mathrm{eff}}=-0.22\right)$ розщеплюється у спектрах плям подібно до ліній з позитивним фактором Ланде $\left(g_{\text {eff }}>0\right)$. Обговорено можливі причини цього ефекту.

Ключові слова: Сонце, сонячні плями, ефект Зеємана, магнітні поля, прямі вимірювання, фактори Ланде.

Вступ. Вимірювання магнітних полів по спектральних лініях з різними факторами Ланде важливі для досліджень магнітних полів, які $є$ неоднорідними у горизонтальній площині. Теоретично при однорідному магнітному полі величина виміряного поля $B_{\text {obs }}$ не повинна залежати від фактора Ланде і має відповідати дійсному магнітному полю $B_{\text {true. }}$ Однак при неоднорідних магнітних полях це не так; зокрема, якщо спостерігається поверхнева неоднорідність магнітного поля у формі поєднання більш слабкого фонового поля і більш сильного поля, зосередженого у маломасштабних (просторово нероздільних) силових трубках, то за певних умов виникає ефект "зеєманівського насичення" [9]. При цьому лінії з різними факторами Ланде можуть давати суттєво відмінні (у кілька разів) виміряні магнітні поля, якщо використовувати традиційні методи інтерпретації спостережень. Перші результати щодо цього були отримані в роботі [1] магнітографічним методом на основі порівняння даних вимірювань в 13 спектральних лініях. Було виявлено, що для слабких магнітних полів за межами сонячних плям ( $\leq 100$ Гc) існує залежність виміряного поздовжнього 